\title{
Are Critical Phenomena Relevant to Large-Scale Evolution?
}

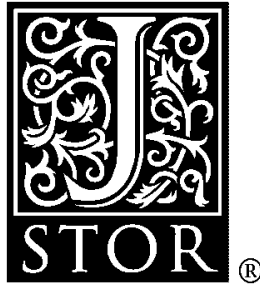

\author{
Ricard V. Sole; Jordi Bascompte \\ Proceedings: Biological Sciences, Vol. 263, No. 1367. (Feb. 22, 1996), pp. 161-168.
}

Stable URL:

http://links.jstor.org/sici?sici=0962-8452\%2819960222\%29263\%3A1367\%3C161\%3AACPRTL\%3E2.0.CO\%3B2-2

Proceedings: Biological Sciences is currently published by The Royal Society.

Your use of the JSTOR archive indicates your acceptance of JSTOR's Terms and Conditions of Use, available at

http://www.jstor.org/about/terms.html. JSTOR's Terms and Conditions of Use provides, in part, that unless you have obtained prior permission, you may not download an entire issue of a journal or multiple copies of articles, and you may use content in the JSTOR archive only for your personal, non-commercial use.

Please contact the publisher regarding any further use of this work. Publisher contact information may be obtained at http://www.jstor.org/journals/rsl.html.

Each copy of any part of a JSTOR transmission must contain the same copyright notice that appears on the screen or printed page of such transmission.

The JSTOR Archive is a trusted digital repository providing for long-term preservation and access to leading academic journals and scholarly literature from around the world. The Archive is supported by libraries, scholarly societies, publishers, and foundations. It is an initiative of JSTOR, a not-for-profit organization with a mission to help the scholarly community take advantage of advances in technology. For more information regarding JSTOR, please contact support@jstor.org. 


\title{
Are critical phenomena relevant to large-scale evolution?
}

\author{
RICARD V.SOLÉ ${ }^{1}$ AND JORDI BASCOMPTE SO, $^{1,2}$ \\ ${ }^{1}$ Complex Systems Research Group, Departament de Fisica i Enginyeria Nuclear, Universitat Politènica de Catalunya, \\ Sor Eulalia d'Anzizu s/n, Campus Nord, Modul B4, 08034 Barcelona, Spain \\ ${ }^{2}$ Department of Ecology and Evolutionary Biology, University of California, Irvine, California 92717, U.S.A.
}

\section{SUMMARY}

Recent theoretical studies, based on the theory of self-organized critical systems, seem to suggest that the dynamical patterns of macroevolution could belong to such class of critical phenomena. Two basic approaches have been proposed: the Kauffman-Johnsen model (based on the use of coupled fitness landscapes) and the Bak-Sneppen model. Both are reviewed here. These models are oversimplified pictures of biological evolution, but the (possible) validity of them is based on the concept of universality, i.e. that apparently very different systems sharing some few common properties should also behave in a very similar way. In this paper we explore the current evidence from the fossil record, showing that some properties that are suggestive of critical dynamics would also be the result of random phenomema. Some general properties of the large-scale pattern of evolution, which should be reproduced by these models, are discussed.

\section{INTRODUGTION}

The large-scale extinction pattern of evolution, as obtained through the analysis of the fossil record, shows several non-trivial trends (see Briggs \& Crowther 1990 and references therein). First, there are some well defined mass-extinction events that are known to be associated with external causes. The fall of an asteroid at the cretaceous boundary, climate changes linked with the dynamics of tectonic plates and other regressional palaeogeographical events are examples of these physically induced catastrophes.

But though these events might have annihilated up to $96 \%$ of species over a short period of time (Raup 1986) they only represent the $5 \%$ of the total extinctions on Earth. In this sense, there is a $95 \%$ of the extinction pattern, the so called background extinctions, which remain to be explained. The first striking fact is that, though we should expect from the two types of extinction events to behave very differently, the study of the frequency distribution of extinction sizes seem to reveal a continuous connection from small to large events, as is shown in figure $1 a$ (Raup 1986).

This result is a bit counterintuitive, as long as a twopeaked frequency distribution should be expected, instead of a fairly continuous one. Other measures, such as the life span of different genera (measured in millions of years, see figure $1 b$ ) also shows a continuous decay. Both curves are relevant in our discussion. Which type of mathematical distribution do they follow? We see a well-defined statistical property of these data: they have a skewed shape (Raup 1993). Many biological data show this type of shape and can be obtained in several ways. A particularly well studied situation is the gambler's ruin problem, where the probability of total ruin for a gambler playing randomly can be calculated. This calculation is done in terms of how many steps are necessary before the ruin takes place (Raup 1993). In terms of extinction, we may think of the gambler's stake as the number of species in an evolutionary group. The resulting distribution of extinctions will be skewed.

In both figures, we have interpolated the available data with an exponential function and with a potential (power law) one. If we indicate by $S$ the extinction size, we have $N(S) \approx S^{-1.95}$ for the potential curve and $N(S) \approx \exp (-0.028 S)$ for the exponential. And for the life span $L$, we get $L$, we get $N(L) \approx L^{-1.84}$ and $N(L) \approx \exp (-0.043 L)$ respectively.

Both curves fit quite well with the data (though the potential one is slightly better). But the dynamical interpetation of them is far from trivial. If a power law (potential) function is involved, then we could conjecture that-at least to some extent-some basic mechanisms operate at different scales. This claim comes from the well-known fact that power-laws are a characteristic of fractal, self-similar objects (Schroeder 1991). To see this, let us take an arbitrary power-law dependent quantity, say $x$. So we have $N(x)=C x^{-\alpha}$, where $C$ is a given constant. If a given scale is considered, say $x^{\prime}=\gamma x$, where $\gamma>0$ defines the change of scale, we have $N\left(x^{\prime}\right)=C\left(x^{\prime}\right)^{-\alpha}$ but by reordering terms, we get in fact $N\left(x^{\prime}\right)=C^{\prime} x^{-\alpha}$ (here $C^{\prime}=C \gamma^{-\alpha}$ ), and so we can extrapolate from a given scale to another. We say that the pattern is self-similar or scaleinvariant. A very different situation is found if an exponential distribution is obtained: scale invariance is absent, and particular characteristic scales are relevant. 


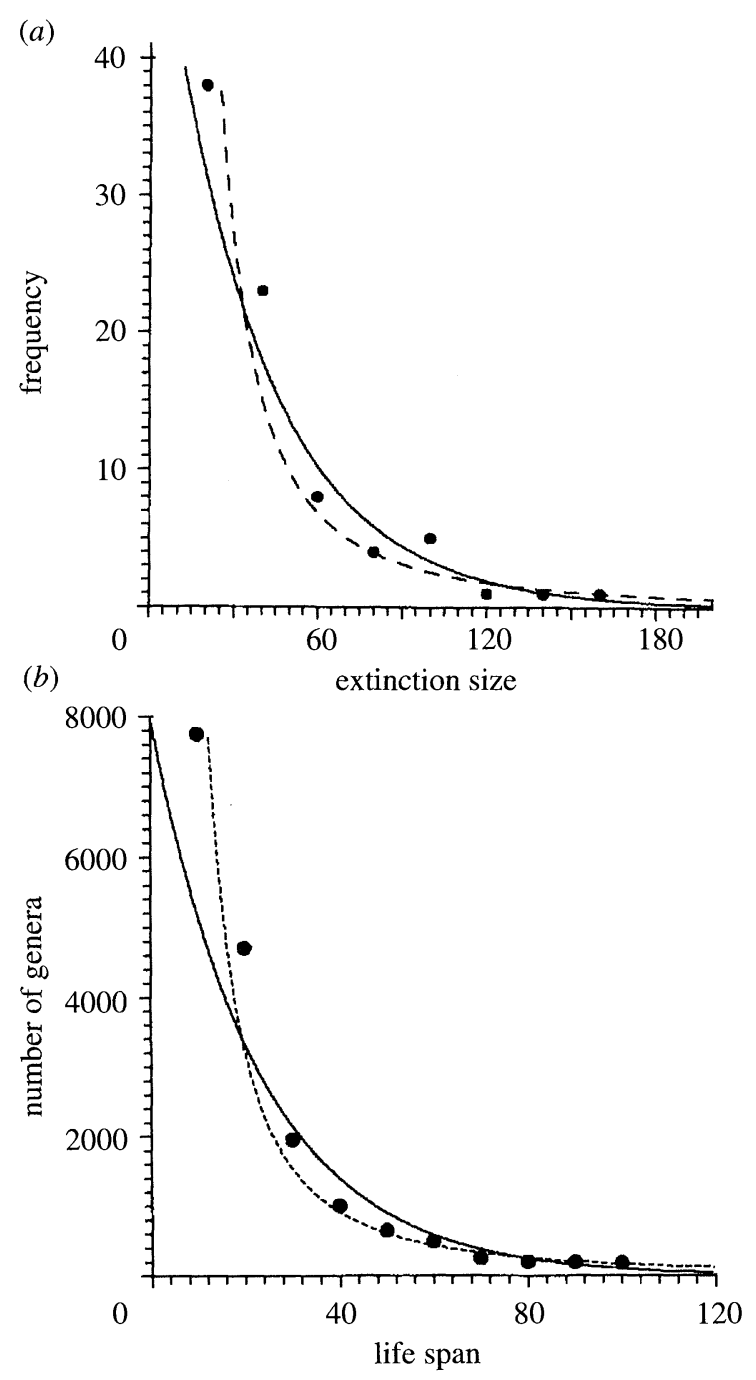

Figure 1. (a) Frequency distribution of extinction sizes for the 79 geologic stages of Phanerozoic time, based on recorded times of extinction of 2316 marine animal families (redrawn from Raup 1986); (b) distribution of geologic life spans of fossil genera (redrawn after Raup 1993). In both figures the exponential (continuous line) and the power law (dashed line) fits to data are shown.

The first situation takes place in all systems poised at critical points. For example, in some magnets when cooled from high temperatures, there is a critical point (the so called Curie temperature) where sudden changes take place. At the critical point, fractal structures spontaneously form, and all relevant quantities show power law, scale free distributions. Those dynamical situations involving phase transitions sharing the previously mentioned properties are generically known as critical phenomena (Binney et al. 1993). The second situation, when exponential-like distributions are involved, is typical for physical systems at equilibrium, when interactions among units are not important and random phenomena are more relevant. Unfortunately, this separation is not so strong. Sometimes, random systems (even a simple random walk) can show some power-law behaviour (Schroeder 1991).

Another trend in evolutionary dynamics is the constant probability of extinction shown by the survivorship curves of most known biological groups.

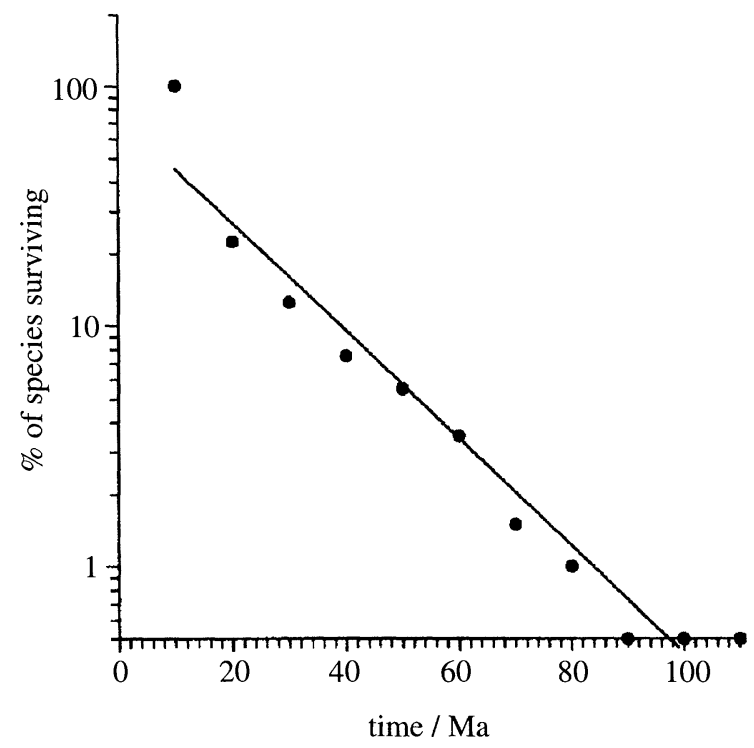

Figure 2. Survivorship of Paleozoic Ammonoidea genus. A nearly constant decay is obtained, well fitted by an exponential curve (see text).

In figure 2, we show such a plot for the planktonic foraminifera (Stenseth 1979; Stenseth \& Maynard Smith 1984).

How is this constancy interpreted? One of the most influential theoretical approaches was first developed by Leigh Van Valen (Van Valen 1973). It is known as the Red Queen hypothesis. The plots of species survivorship shows us that, contrary to our expectations, the probability of extinction within any group remains basically constant through time. A species might disappear at any time, irrespective of how long it has already existed. We should expect species within a group to become longer-lived over time on average. The Van Valen's approach to this problem was a strongly dynamical view of evolution. The basic idea is that species in ecosystems are in a constantly evolving interaction.

Both random-like phenomena and deterministic, dynamical causes are apparently consistent with the qualitative trends of macroevolution. Additionally, punctuationist biologists (see Gould \& Eldredge 1993, and references therein) claim that micro- and macroevolution can be decoupled. In this sense, the features upon which selection acts are those of entire species populations, which are irreducible to the level of individuals (see Skelton 1985). The basic proposal comes from the interpretation of the fossil record as the interplay of long periods of little evolutionary change (i.e. stasis) punctuated by spells of fast change. These rapid changes are linked with species diversification.

\section{GRITIGALITY AND MAGROEVOLUTION}

A macroscopic long-term theory of evolution is a challenging problem. Different fields, as genetics and ecology, should be integrated (if possible) and a coherent picture of extinction and diversification could then emerge. This is at least what some evolutionary biologists expect (for a review of this debate, see 
Stenseth 1985). But some general questions are open. If punctuationists are right, then no general theory of macroevolution can be built from population genetics. We could say, following Philip Anderson, that macroevolution is not applied population genetics.

Recently, a new theoretical framework has been proposed by some researchers (Kauffman \& Johnsen 1991 ; Bak \& Sneppen 1993). The underlying approach comes from the theory of self-organized critical phenomena (Bak et al. 1989). Bak and his colleagues analysed a striking result coming both from physical and biological systems: the widespread presence of fractal structures in nature (Sugihara \& May 1990). Fractal, free-scale objects are observed at all scales of biological organization, from cells and organs (Goldberger et al. 1990) to rainforests (Solé \& Manrubia $1995 a, b)$. Though the theory of fractal objects was highly developed, no theory of how these objects originate has been proposed since the BTw paper (Kadanoff 1986).

As mentioned in the previous section, physical systems poised at critical points show fractal behaviour. This suggested to Bak an appealing possibility: perhaps those systems displayed scale-free properties were in fact the result of a self-organization process. Following this idea, they coined the concept of self-organized criticality (soc). Self-organized critical systems are able to drive themselves towards the phase transition points (called 'the edge of chaos' by some authors, see Kauffman 1992). Sandpiles, earthquakes and developed turbulence seem to be good candidates of soc systems.

Are biological systems poised at a critical state? The answer to this question, if affirmative, would be of enormous importance. If soc dynamics are involved, then a continuous, scale-free connection between small and large events naturally emerges. Some recent studies involving the dynamics of tropical rainforests (where several field data are available) seem to give support to this possibility, at least at some scales (Solé \& Manrubia $1995 a, b)$.

In relation to evolution, criticality implies that large extinctions are likely to occur, being generated by the same mechanism as small extinctions. In this context, extinctions should be considered as an unavoidable and intrinsic part of the macroevolutionary dynamics. Thus, if somebody waits long enough, they are bound to see events that are as large as they had the patience to wait for (Bak \& Paczuski 1995). This is one of the claims of Bak \& Kauffman. These models have generated some controversy (Maddox 1994, 1995). A very important discussion is concerned with how to test these models as well as how to separate random effects from other deterministic phenomena. In the next section we review some basic properties and predictions of soc models.

\section{THEORETIGAL MODELS}

\section{(a) Kauffman-Johnsen Model (кJM)}

Kauffmann and Johnsen start with a version of the well-known NK fitness landscapes (Kauffman \& Levin 1987). Here $N$ stands for the number of binary elements or 'genes' which characterize the particular traits of a 'species'. These units are coupled among them by means of a finite (fixed) number $1 \leqslant K \leqslant N$ of connections, which gives a measure of the richness of epistatic interactions among genes. Though a fixed connectivity $K$ can seem a very restrictive constraint, it can be relaxed to more general situations, where distributions of $K$ values are used (Solé \& Luque 1995).

For this model, the fitness landscape is defined by assigning to each possible configuration of connected genes a given random number (the fitness). A simple dynamical rule is applied: we take a given configuration (a string of $N$ binary units) and we switch at random a single unit. Then we compute the new fitness $\omega$. If the change increases $\omega$ it is accepted (an adaptive walk is done) if not, it is rejected (Maynard Smith 1970; Kauffman \& Levin 1987). The next step is to consider a set of $S$ species, each defined through an $N K$ network, and couple them. To this end we consider $C$ new interspecific couplings. Then $0<C<N$ additional connections are made at random between the units of each species and $C$ units of other species. So now adaptive walks of a single species modify the biotic environment of the other $S-1$ species: coevolution occurs. Typically, the system evolves towards a kind of Nash equilibria: species reach local optima (Kauffman \& Johnsen 1991). Finally, a source of external perturbations is used (the external environment) by adding some additional couplings. When such coupling is introduced, the external perturbations (involving a small change each time) can propagate through the system. Few, several or many changes can occur before a new Nash equilibria is reached. The number of changes defines the size of the so called 'evolutionary avalanche'. Measuring the frequency of such avalanches, a power law can be obtained (Kauffman \& Johnsen 1991; Kauffman 1992). An additional trait of the dynamics of the NKG model is that periods of 'stasis' (with small avalanches) are interrupted by coevolutionary avalanches.

\section{(b) Bak-Sneppen model (BSM)}

The Bak-Sneppen model is defined by a simple set of rules (Bak \& Sneppen 1993). It is a very simple metaphor of an evolving ecosystem, and can be easily explained. We consider a string of $N$ random numbers $x_{i} \in(0,1)$, which are the 'species' of our ecosystem, and the steps are: (i) we choose the minimum $x_{j}$ and change it by a new random number $x_{j} \in(0,1)$; (ii) the two nearest neighbours are also changed: $x_{j+1} \in(0,1)$, $x_{j-1} \in(0,1)$ (periodic boundaries are assumed); and (iii) the previous steps are repeated.

This is a simple model of a set of species evolving in a fitness landscape. In this model the quantities $\left\{x_{i}\right\}$ play the role of a 'barrier height', separating the local fitness maximum from other maxima. In other words, the barrier height is a measure of how far is the $i$ th species from its maximum fitness. Then if the fitness $x_{j}$ is low, it is more likely to find nearby better states. In the BSM, the barriers are the measure of stability: smaller barriers are more unstable and easily changed. In spite of the oversimplified nature of this model, it is 
able to show punctuated equilibrium in terms of bursts of change into the ecosystem. It also shows power law distributions of several quantities. These properties are in fact shared by other complex systems as economics, and it has been conjectured that punctuated equilibrium is a common property of all complex systems.

Both models involve changes at the species level (Maddox 1994) instead of at the level of individuals, which is what we expect selection to act upon. This is linked with the timescale under consideration, and has been used in other earlier theories. Maynard Smith \& Stenseth have distinguished among three timescales, the largest one being the so called speciation-extinction timescale (Stenseth 1985). Here the number of coexisting species becomes variable, and theories for the behaviour of the fossil record refer to this timescale. If universal properties are involved, the specific details of the elements and their interactions becomes less relevant. This could justify this approach based upon a higher level of interaction.

\section{1/f-DYNAMICS AND RANDOMNESS}

A characteristic fingerprint of complex systems poised at a critical state is provided by the observation of a widely extended type of temporal self-similarity: the so called one-over-f $(1 / f)$ noise. These type of fluctuations are characterized by a power spectrum $P(f)$ with a power-law distribution, $P(f) \approx f^{-\beta}$ with $\beta \approx 1$. Though $1 / f$-fluctuations are detected in the BsM, as far as we know there is no study of real data involving the analysis of this property.

In figure $(3 a)$, we show the fluctuations, over $320 \mathrm{Ma}$ of family diversity of the Ammonoidea (House 1989), shown from the early Devonian to the end of Cretaceous (when they became extinct). The corresponding Fourier spectrum (Fs) was calculated, and it is shown in figure $(3 b)$. Interestingly, it is not far from the $1 / f$ spectrum. We have found an exponent $\beta=0.87$. Though the time series is small, and the Fs quite noisy, this seem to give support to the conjecture of critical states. But as mentioned in previous sections, power laws can also be present in random-like processes. So we have to explore whether or not a similar pattern would be obtained from a random process. Let us consider two examples.

We have plotted in figure $(3 c)$ a sample of a computer simulation of a random walk. Here we start with $N(0)=1$. The rules are very simple. At each time step $t$, a random number is generated (with uniform distribution) $x_{i} \in(0,1)$. If $x_{i}<0.5$, we add a unit to $N(t)$ otherwise, we subtract one unit. The only restriction is that $N(t)$ cannot be less than one (if $N(t)=1$ and $x_{i}>0.5$, we take $N(t+1)=1$ ) (i.e. we have a reflecting boundary). Then let us take a random sample which look similar to the Ammonoidea time series (figure $(3 c)$ ). Though it is well known that the random walk with no restrictions exhibits $1 / f^{2} \mathrm{dy}$ -
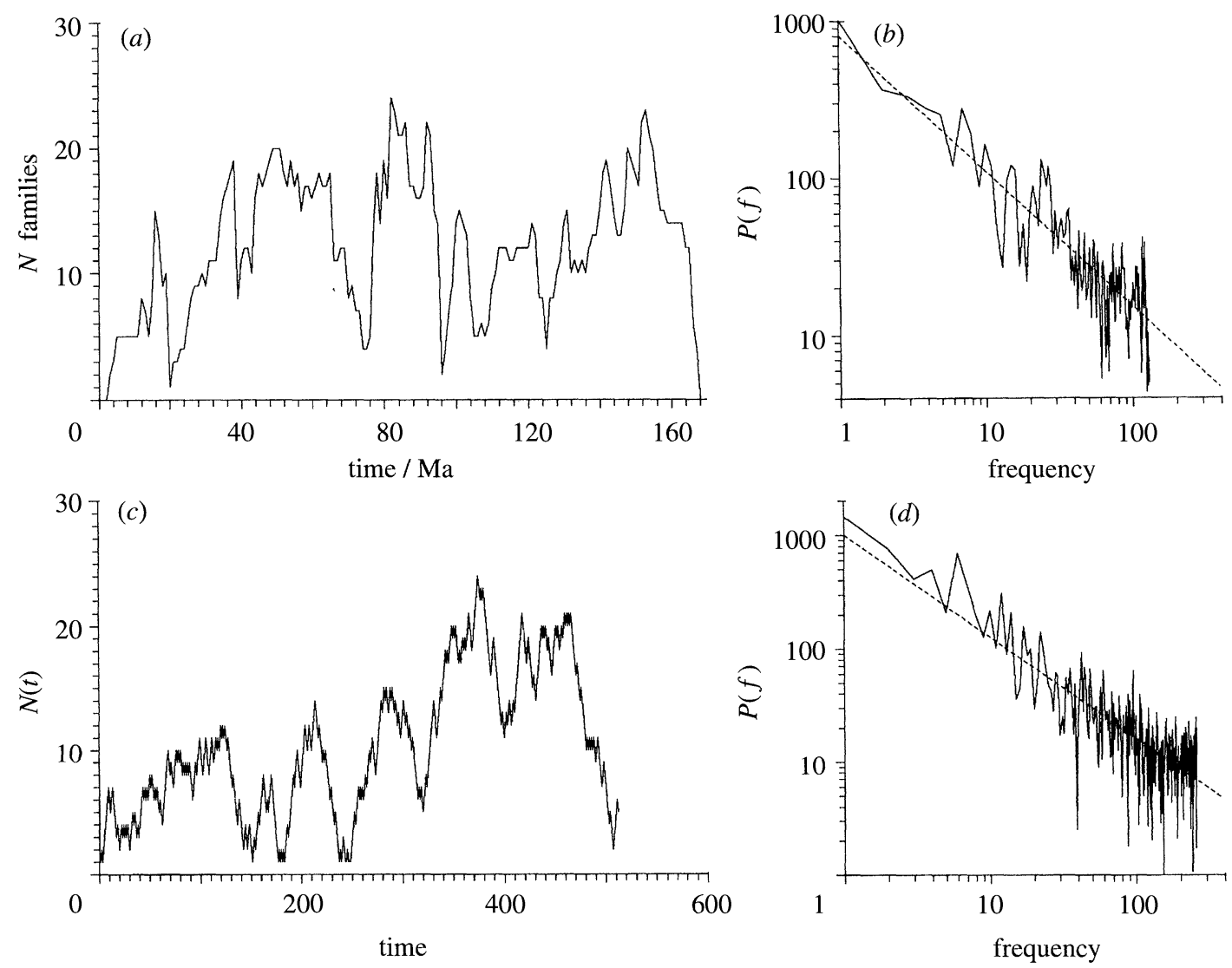

Figure 3. (a) Fluctuations of Ammonoidea family numbers over time. Here the scale is $\times 2 \mathrm{Ma}$, over a period of $320 \mathrm{Ma}$ (redrawn from House 1989); the corresponding Fourier spectrum is shown in $(b)$, with a power-law shape. (c) Time fluctuations of a random walk with a reflecting boundary at $N=1$. The Fourier spectrum for this sample is shown in $(d)$. 


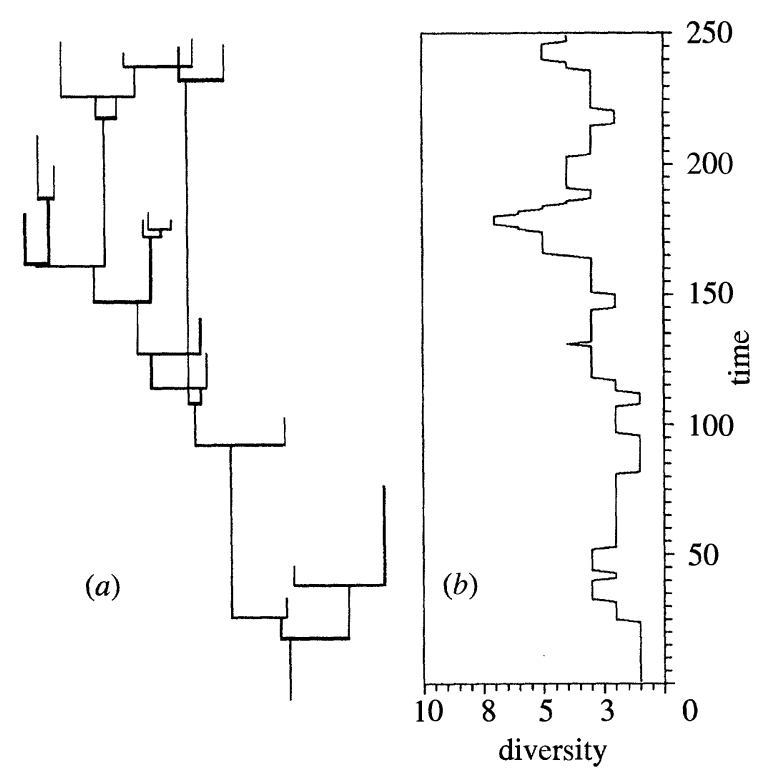

Figure 4. (a) Random phylogenetic tree obtained following the general approach by Raup (Raup 1985). The corresponding dynamics of the diversity of lineages is shown in (b) (see text).

namics (Peitgen \& Saupe 1988; Shroeder 1991) we cannot be sure of what kind of Fs will be obtained from this finite-time simulation. The resulting $P(f)$ is shown in figure $(3 d)$. For this particular sample, we have $\beta=0.87$, close to the Ammonoidea spectrum. Is the Ammonoidea time series a random walk?

Let us finally consider a further model, more close to the natural pattern of evolution. It is described by the well-known mathematical models of cladogenesis (Raup 1985; see also Skelton 1993). These are models of clade dynamics i.e. of the processes of lineage branching and termination, leding to phylogenetic trees. They are very simple. We start from a single lineage, which can become extinct with some probability $P_{e}$ or can persist to the next time step. Then it can branch to produce a second lineage (with some probability $P b$ ) or persist without branching. So both extinction and diversification are involved. A simple example of this phylogeny is shown in figure $(4 a)$ together with the evolution in time of diversity, measured as the number of lineages (figure $(4 b)$ ). If speciation and extinction occurs at similar rates, it is not difficult to obtain fluctuations similar to those from the Ammonoidea time series. An example is shown in figure $5 a$, together with the corresponding Fourier spectrum. For this sample, we get $\beta \approx 0.97$. Again, a random model can lead to an apparently self-organized phenomenon. Can the Ammonoidea time series be explained from a random model of cladogenesis dynamics?

These results are fairly typical. So a direct consequence is that, though the time series of evolution of family diversity of Ammonoidea is quite suggestive of soc, it could be also the result of a random process. Other additional measures can be used, also derived from the previous data (House 1989). If we plot the frequency distribution in the appearance of new families, as well as the extinct ones, we also find power laws, i.e. $N(S) \approx S^{-\eta}$, which are slightly better than the exponential fit (see figure $(6 a, b))$. An important difference is present: the exponent for the power law of extinction sizes is rather different from the previous one $(\eta \approx 1.95)$, based on the recorded times of extinction of 2316 marine animal families (Raup 1986). Now we have $\eta \approx 1.35$. The slope for the new families is $\eta \approx$ 1.85. So the slope for the whole recorded marine families is not the same as the one for a particular group (the Ammonoidea) assuming a power-law fit. This is an important point for further theoretical models based on soc theories. It suggests that more detailed data, involving different scales, have to be taken into account.

Are power-laws also obtained from the previous model of cladogenesis? By following through time the branching of these cladograms, we can compute the frequency distribution of new branches as well as the extinct ones. The answer is negative. All our simulations have shown that the resulting distributions are exponential or even Gaussian-like. So even if a $1 / f$-like dynamics can be observed when short time series are studied, some other statistical properties behave in a very different way. So we have obtained an interesting result: the time evolution and the extinction-
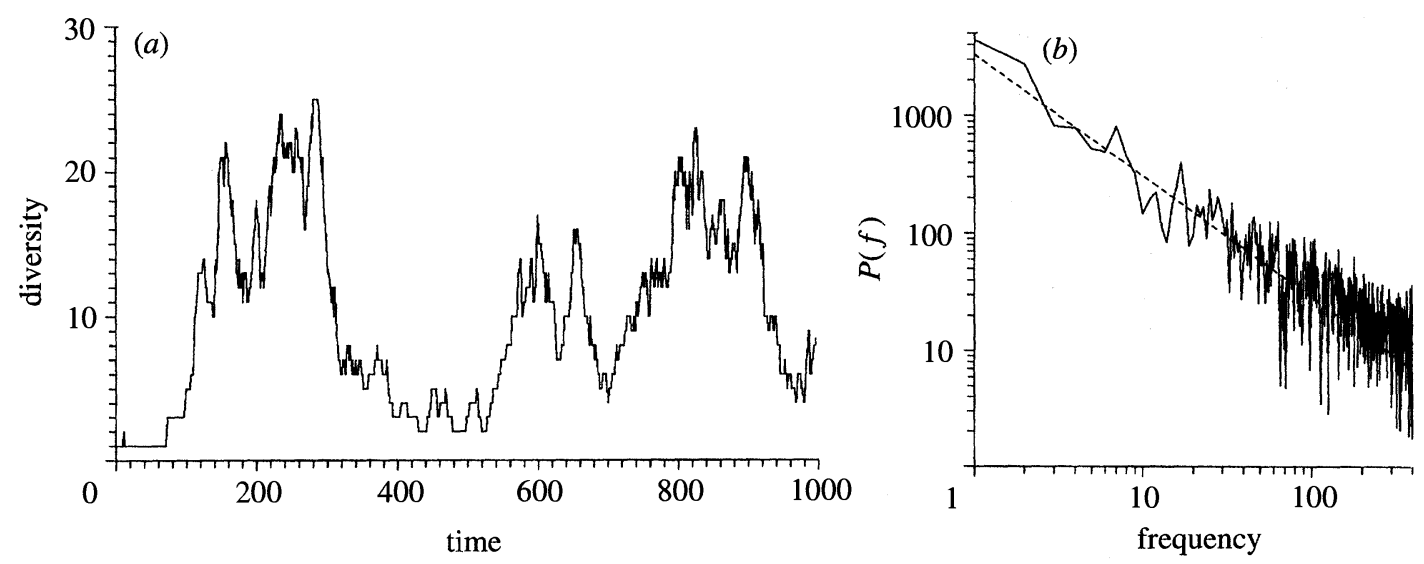

Figure 5. (a) Time fluctuations of diversity in a random model of phylogenetic dynamics (see figure 4). Here the branching probability is $p_{b}=0.40$ and the extinction probability (of a lineage) is $p_{e}=0.38$. In $(b)$ the corresponding Fourier spectrum is shown. 
(a)

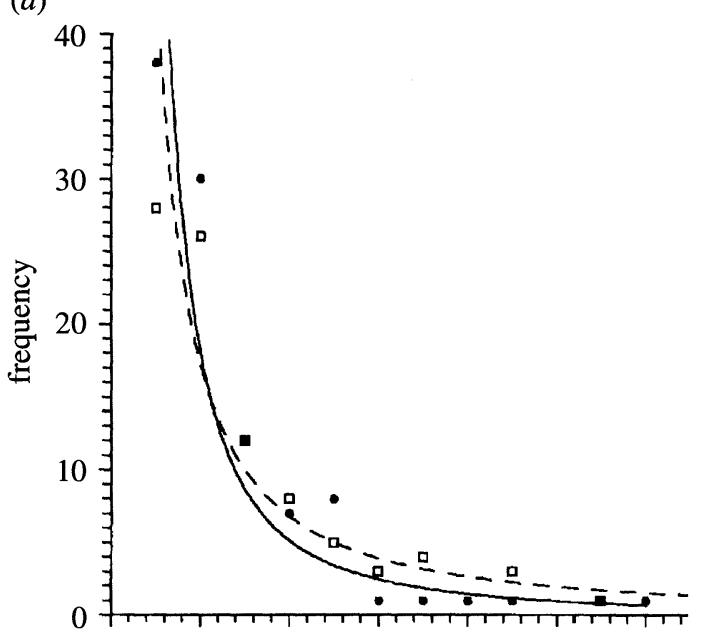

(b)

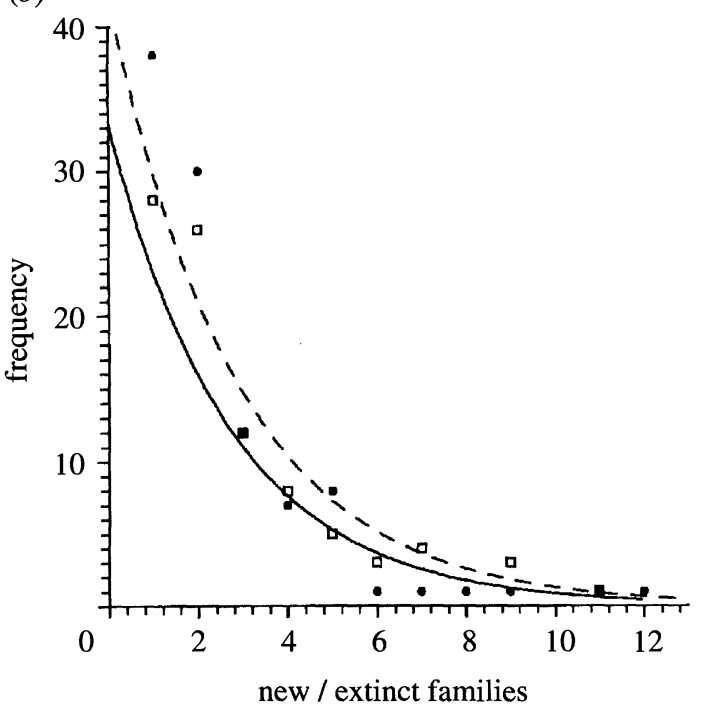

Figure 6. Frequency distribution of new (filled symbols) and extinct (open symbols) Ammonoidea families (see text). In (a) a power-law fit has been used; in $(b)$ an exponential fit has been used.

diversification pattern both seem compatible with a self-organized critical phenomenon.

\section{THE FRAGTAL NATURE OF TAXONOMY}

There is a further evidence of power-law behaviour in the dynamics of macroevolution. This evidence comes from the fractal properties underlying taxonomic systems (Burlando 1990, 1993). In a series of remarkable studies both from living and fossil taxa, it has been shown that the numbers of subtaxa $(S)$ within fossil taxa $(T)$ (families within orders, species within genera, etc) are organized (now very clearly) in a power-law manner, i.e. $T(S) \approx S^{-\gamma}$. Similar values occur for the same group in taxonomic lists from distant world areas, usually studied by different taxonomists. The finding that similar exponents are observed at different taxonomic levels supports the idea of a free-scale phenomenon underlying evolution. These are probably the most relevant data sets supporting a critical phenomenon. As stated by
Burlando, this arrangement of life taxonomy leads to the conclusion that such a pattern reflects evolutionary features (see also Green 1991). It is important to mention that a scaling pattern has never been considered in evolutionary studies, though the results of Burlando strongly support the presence of selfsimilarity in the dynamics of evolution. A recent study, based on a derivation of the BSM has shown that selfsimilar phylogenetic trees can be generated (Vandewalle \& Ausloos 1995) from a self-organized critical process. This is not what we obtain from a random model of phylogeny. As a consequence, fractal taxonomic patterns are probably the result of a nonrandom process but a critical one.

\section{DISGUSSION}

In this paper we have reviewed some of the basic ideas underlying the theoretical approaches to macroevolution based upon the theory of self-organized critical phenomena. We have seen that some data from the fossil record, such as the distribution of extinctions, seem to suggest the existence of power laws. That is what should be expected in systems poised at a critical state. But these data are not conclusive. They could be the result of random processes. Other data, as those obtained from the study of the taxonomy, shows that a fractal organization of taxonomic trees is present. These studies cannot be trivially interpreted from random processes.

In table 1, the previous points discussed through this paper are summarized. Different aspects of the macroevolutionary pattern together with the observed trends are given. What should be expected from a random process and from a soc state is also outlined. These properties have been discussed in previous sections. The field evidence analysed by Bak and his colleagues (Sneppen et al. 1995; Bak \& Paczuski 1995) and by Kauffman \& Johnsen (Kauffman \& Johnsen 1991) is mostly based upon the statistical properties of extinction patterns and life spans. Though a power law can be well fitted to these data sets, the fit is also correct for an exponential distribution. The analysis of the dynamical evolution of Ammonoidea families performed in this paper also shows evidence of temporal

Table 1. Some basic trends of macroevolutionary patterns

\begin{tabular}{|c|c|c|c|}
\hline property & observed & $\begin{array}{l}\text { random } \\
\text { process }\end{array}$ & SOC \\
\hline $\begin{array}{c}\text { extinction } \\
\text { pattern }\end{array}$ & skewed $^{\mathrm{a}}$ & exponential & power-law \\
\hline $\begin{array}{l}\text { dynamics } \\
\text { (rate) }\end{array}$ & punctuated & $\begin{array}{l}\text { random } \\
\text { walk }\end{array}$ & punctuated \\
\hline $\begin{array}{l}\text { dynamics } \\
\text { (time) }\end{array}$ & $1 / f^{\mathrm{b}}$ & $1 / f^{2}$ & $1 / f$ \\
\hline $\begin{array}{l}\text { taxonomy } \\
\text { mass } \\
\text { extinctions }\end{array}$ & $\begin{array}{l}\text { self-similar } \\
\text { few events }\end{array}$ & $\begin{array}{l}\text { non-fractal } \\
\text { virtually } \\
\text { impossible }\end{array}$ & $\begin{array}{l}\text { self-similar } \\
\text { expected }\end{array}$ \\
\hline
\end{tabular}

a Available data compatible both with exponential and power-law fits, with better agreement with the second distribution.

b Based only in the Ammonoidea data studied in this paper. 
self-similarity. But a further exploration of models based on random rules can lead to similar results, though some data (as the new-extinct family distributions of Ammonoidea) together with $1 / f$-dynamics, are more consistent with soc dynamics.

It is our belief that some general facts should be taken into account in further models of large-scale evolution.

1. Though the biotic environment is extremely important for the biological organization, a species might go extinct for different reasons, both biotic and physical (external). There is little doubt that the external, physical environment, has played a prominent role. This coupling with the external world is considered in the Kauffman (NKC) model (Kauffman 1984) but not in the BSM. Future models have to test how important is the introduction of such a type of coupling in relation to the strictly biotic interactions (see the work by Newman \& Roberts $1995 a, b$, for an excellent discussion). In this context we have to mention that small amounts of external 'noise' (i.e. the extinction of single species) could in fact induce phase transitions. These are known as noise-induced phase transitions and play a very important role in many physical and biological systems (Horsthemke \& Lefever 1977; Solé \& Luque 1995). In particular, power-laws would result from such a noise-induced critical phenomenon.

2. Evolution takes place at different scales in space and time. Large extinctions are likely in those models exhibiting soc (and by means of identical mechanisms as the small ones). On the other hand, the largest extinction events (so called mass-extinctions) are convincingly associated with global changes. But the interplay between the external effect and the response of the species network is clearly nonlinear. It is very important to understand which scales are linked with criticality (i.e. with linear interactions) and which with random events.

3. We need a theory where both extinction and diversification should be involved (Maddox 1994; Solé et al. 1996). There is an enormous amount of data on biological diversity at many scales. Diversity is a well defined concept in ecology and many data should be used as a source of validation of soc models. An additional phenomenon (as stressed by Maynard Smith 1989 ) is a surprisingly constant diversity (as measured by numbers of genera or families) which has been reported within particular taxa over many millions of years, despite continued extinction and speciation. At a smaller timescale, tested in actual ecosystems, biological diversity is bounded and related with network connectivity (May 1972). In fact, selforganized critical patterns have been recently detected from the study of rainforest ecosystems (Solé \& Manrubia, 1995 $a, b)$. Those events, extinction and diversification, would be linked with the generic stability properties of network ecosystems (May 1972; Solé et al. 1992; Bascompte \& Solé 1993) and so soc models should benefit from these studies.

To sum up, the simple models introduced by Kauffman \& Bak have a potential power for explaining one of the most fascinating problems of evolution: the nature of patterns of extinction and diversification. The underlying hypothesis is that in systems exhibiting soc, the details of the underlying rules are rather irrelevant. Once the key ingredients are introduced, the dynamical outcome is quite universal. Here universality (a well known concept in the theory of critical phenomena; see Binney et al. 1993; Solé et al. 1996) means that the slope of power laws, which characterize the macroscopic pattern (and the basic laws) are the same. This result, confirmed by several models (consistent with real data) is rather important in our discussion. Though more detailed models would be of interest, simplicity is desirable and could be fully justified. The challenge of understanding macroevolution deserves new types of theoretical approaches such as those conjectured by self-organized criticality.

We thank Brian Goodwin, M. E. J. Newman and Robert May for their useful comments. We also thank Per Bak for sending us preprints. This work has been supported by grants of UPC PR9407 and DGYGIT PB94-1195. J.B. is supported by a postdoctoral grant of the Spanish Ministry of Education and Science.

\section{REFERENGES}

Bak, P., Tang, C. \& Wiesenfeld, K. 1989 Self-organized criticality: an explanation for $1 / f$ noise. Phys. Rev. Lett. 59, 381-384.

Bak, P. \& Sneppen, K. 1993 Punctuated equilibrium and criticality in a simple model of evolution. Phys. Rev. Lett. 4083-4086.

Bak, P. \& Paczuski, M. 1996 Complexity, contingency and criticality. Proc. natn. Acad. Sci. U.S.A. (In the press.)

Bascompte, J. \& Solé, R. V. 1993 Diversity at the edge of chaos; a new look at the barroque of nature. In Proceedings of the 1st Copenhagen Symposium in comp. Sim. Biol. Ecol. Med. (ed. E. Mosekilde) pp. 56-60.

Binney, J.J., Dowrick, N. W., Fisher, A.J. \& Newman, M. E. J. 1993 The theory of critical phenomena. Oxford: Clarendon Press.

Briggs, D. E. G. \& Crowther, P. R. (eds) 1990 Palaeobiology. Oxford: Blackwell.

Burlando, B. 1990 The fractal dimension of taxonomic systems. J. theor. Biol. 146, 99-114.

Burlando, B. 1993 The fractal geometry of evolution. $J$. theor. Biol. 163, 161-172.

Goldberger, A. L., Rigney, D. R. \& West, B. J. 1990 Sci. Am. 241, 34-41.

Gould, S. J. \& Eldredge, N. 1993 Punctuated equilibrium comes of age. Nature, Lond. 366, 223-227.

Green, D. M. 1991 Chaos, fractals and nonlinear dynamics in evolution and phylogeny. Trends Ecol. Evol. 6, 333-337.

Horsthemke, W. \& Lefever, R. 1984 Noise-iduced transitions. Berlin: Springer-Verlag.

House, M. R. 1989 Ammonoid extinction events. Phil. Trans. R. Soc. Lond. B 325, 307-326.

Jablonski, D. 1987 Mass and background extinctions: the alternation of macroevolutionary regimes. Science, Wash. 231, 129-133.

Kadanoff, L. P. 1991 Fractals: where is the physics? Phys. Tod. 39, 6-7.

Kauffman, S. A. \& Levin, S. 1987 Towards a general theory of adaptive walks on rugged landscapes. J. theor. Biol. 128, 11-45.

Kauffman, S. \& Johnsen, J. 1991 Coevolution to the edge of chaos: coupled fitness landscapes, poised states and coevolutionary avalanches. J. theory. Biol. 149, 467-505. 
Kauffman, S. 1992 The origins of order. Oxford University Press.

Maddox, J. 1994 Punctuated equilibrium on a computer. Nature, Lond. 371, 197.

Maddox, J. 1995 Polite row about models in biology. Nature, Lond. 373, 555.

May, R. M. 1972 Will a large complex system be stable? Nature, Lond. 238, 413-414.

Maynard Smith, J. 1970 Natural selection and the notion of protein space. Nature, Lond. 225, 563-566.

Maynard Smith, J. 1989 The causes of extinction. Phil. Trans. R. Soc. Lond. B 325, 241-252.

Newman, M. E. J. \& Roberts, B. W. 1995 a Mass extinction: evolution and the effects of external influences on unfit species. Proc. R. Soc. Lond. B 260, 31-37.

Newman, M. E. J. \& Roberts, B. W. $1995 b$ A model for evolution and extinction. Technical Report CTC 95TR220. Cornell University Press.

Peitgen, H. \& Saupe, D. (eds.) 1988 The science of fractal images. New York: Springer-Verlag.

Raup, D. M. 1985 Mathematical models of cladogenesis. Paleobiology 11, 42-52.

Raup, D. M. 1986 Biological extinction and Earth history. Science, Wash. 231, 1528-1533.

Raup, D. M. 1993 Extinction: bad genes or bad luck? Oxford University Press.

Schroeder, M. 1991 Fractals, chaos and power laws. New York: Freeman and Co.

Skelton, P. (ed.) 1993 Evolution. Wokingham: AddisonWesley.

Sneppen, K., Bak, P., Flyvbjerg, H. \& Jensen, M. H. 1995 Evolution as a self-organized critical phenomenon. Proc. natn. Acad. Sci. U.S.A. 92, 5209-5213.
Solé, R. V., Bascompte, J. \& Valls, J. 1992 Nonequilibrium dynamics in lattice ecosystems: chaotic stability and dissipative structures. Chaos 2, 387-395.

Solé, R. V. \& Manrubia, S. C. 1995 a Are rainforests selforganized in a critical state? J. theor. Biol. 173, 31-40.

Solé, R. V. \& Manrubia, S. G. $1995 b$ Self-similarity in rainforests: evidence for a critical state. Phys. Rev. E 51, 6250-6253.

Solé, R. V. \& Luque, B. 1995 Phase transitions and antichaos in generalized Kauffman networks. Phys. Lett. A 196, 331-334.

Solé, R. V. 1996 On macroevolution, extinctions and critical phenomena. Complexity (In the press.)

Solé, R. V., Manrubia, S. G., Luque, B., Delgado, J. \& Bascompte, J. 1996 Phase transitions and complex systems. Complexity (In the press.)

Stenseth, N. G. 1985 Darwinian evolution in ecosystems: the Red Queen view. In Evolution. Cambridge University Press.

Stenseth, N. C. \& Maynard Smith, J. 1984 Coevolution in ecosystems: red queen evolution or stasis? Evolution 38, 870-880.

Sugihara, G. \& May, R. M. 1990 Application of fractals in ecology. Trends Ecol. Evol. 5, 79-86.

Vandewalle, N. \& Ausloos, M. 1995 Self-organized criticality in phylogenetic-like tree growths. J. Phys. I France 5, 1011-1025.

Van Valen, L. 1973 A new evolutionary law. Evol. Theory 1, $1-30$.

Received 8 August 1995; accepted 14 November 1995 